\title{
Comparative Study of Cross and Lateral Fixations on Supracondylar Humerus Fracture among Children
}

\author{
Komang Agung Irianto, Tri Wahyu Martanto, Febrian Brahmana, Laskar PK
}

Department Orthopedics and Traumatology, Faculty of Medicine, Universitas Airlangga/ Dr. Soetomo Hospital, East Java

\section{ABSTRACT}

Background: Management of Gartland type III supracondylar humerus fractures is conducted by open and closed repositioning. An adequate reposition and a stable and accurate fixation are desperately needed to prevent fixation failure, deformity, and complication. The study aims to compare the clinical and radiological result between crossed and lateral fixation techniques.

Subjects and Method: The study was a retrospective study toward Gartland type III SCHF children in Dr. Soetomo Hospital, Surabaya, Indonesia from 2013-2016. The dependent variable is Supracondylar humerus fracture. Independent variables is type of fixation option, clinical functional test, degrees of satisfactory, and radiology evaluation. The radiology parameter used was Skaggs criteria. An observation was conducted for the occurrence of complication in the form of infection and peripheral nerves injury. All data were analyzed using Kolmogorov Smirnov and Fischer exact test.

Results: The study discovered 28 patients consisted of 20 males and 8 females with age range from $3-13$ years old with average age in crossed fixation group was 7.6 years and in lateral fixation was 4.7 years. The injury sides were $46.4 \%$ right elbow and $53.5 \%$ left elbow. Among the crossed fixation group there were 54.5 \% left elbow and 45.5\% right elbow. Among lateral fixation group there were $50 \%$ left side and 50\% right side. There was no significant different on clinical functions, radiology as well as complication in the form of infection and peripheral nerves injury.

Conclusion: There is no difference of functional clinical, radiology result as well as post-surgery complication in the form of infection and peripheral nerves injury between crossed fixation technique and lateral fixation technique.

Keywords: Supracondylar Humerus Fracture, K-wire fixation, K-wire, Lateral Approach, Complication

\section{Correspondence:}

Komang Agung Irianto. Department of Orthopedics and Traumatology, Faculty of Medicine, Universitas Airlangga/ Dr. Soetomo Hospital, Surabaya. Email: komang168@yahoo.com. Mobile: +62811336080 .

Cite this as:

Irianto KA, Martanto TW, Brahmana F, Laskar PK (2020). Comparative Study of Cross and Lateral Fixations on Supracondylar Humerus Fracture among Children. Indones J Med. 05(01): 31-37.

https://doi.org/10.26911/theijmed.2020.05.01.05

cc) (i) (2) Indonesian Journal of Medicine is licensed under a Creative Commons Attribution-Non Commercial-Share Alike 4.o International License.

\section{BACKGROUND}

Supracondylar humerus fracture often happens to children, it is the $2^{\text {nd }}$ most common fracture after antebrachii fracture. The worldwide incidences are around 308/100,000 per year. It is increasing along with the age, and hits the highest point at the age of $5-8$ years old, afterward it is decreasing after the age of $8-15$ years old.

According to Gartland (1959) the fracture is classified into non-displaced fracture (type I), displaced fracture with intact posterior cortex (type II), and completely displaced fracture (type III). Three displaced supracondylar humerus fractures are fractures 
which are difficult to be reduced and risk of reduction failure therefore the management approach is relatively challenging for orthopedists (Fransworth et al., 1998; Lins et al., 1999; Gartland 1959; Lee, 2000).

Fixation with K-Wire following supracondylar fracture reduction toward children was firstly introduced by Casino (1960) it is an effective therapy in maintaining reduction. Since then the fixation with K-Wire post supracondylar fracture reduction has become standard therapy for supracondylar humerus fracture among children. However research by Otsuka and Kasser (1997) finding there are complication related to K-Wire fixation including iatrogenic injury of ulnar nerve and reduction failure, that lead to cubitus varus deformity. Research by Gordon et al (2001) and Kalenderer et al. (2008) finding there are an optimal K-Wire configuration gives adequate fracture stability following the reduction and minimizes the risk of reduction failure.

There are several techniques of K- wire fixation configuration for supracondylar humerus fracture, among others are crossed and lateral techniques. Crossed technique is biomechanically more stable however, the focal point is directed to the risk of iatrogenic injury of ulnar nerve during K-Wire medial insertion (Rasool, 1998; Wang et al., 2012). The lateral technique may avoid the risk of iatrogenic injury of ulnar nerve, however the configuration may be less stable (Kocher et al., 2007). The study aims to evaluate the effectiveness of fixation of both crossed fixation and lateral fixation techniques from the clinical and radiology results point of view as well as the occurred complication.

\section{SUBJECTS AND METHOD}

\section{Study Design}

This was a retrospection study that aimed to compare clinical functional and radiological result between crossed fixation and lateral fixation techniques on supracondylar humerus fractures among children in Dr. Soetomo Hospital, Surabaya, Indonesia within 3 years, started from 2013-2016.

\section{Population and Sample}

The study sample was all Gartland type III SCHF that undergone closed reposition procedure and K-Wire fixation with C-Arm guidance. All procedures were performed by on duty orthopedic surgeons in emergency unit at the period of time.

\section{Study Variables}

The dependent variable is Supracondylar humerus fracture. Independent variables is type of fixation option, clinical functional test, degrees of satisfactory, and radiology evaluation.

\section{Operational Definition of Variables} The option of fixation types was determined by on duty seniors at the period of time. Clinical functional test was referred to the passively shifting of conjoint moving space and carrying angle.

Degrees of satisfactory was degree shifting of conjoint moving space and carrying angle. The data scale is categorical with excellent $=0-5$ degrees, good $=5-10$ degrees, fair $=10-15$ degrees. Shifting of conjoint moving space and carrying angle than 15 degrees was categorized as poor (Unsatisfactory).

Radiology evaluation was determined in the form of plain image of Baumann angle according to Skaggs criteria. The shifting of Baumann angle $<6$ degrees was categorized as none, 6-12 degrees was categorized as mild and major if it was more than 12 degrees.15 Complications in the form of surgical wound infection and peripheral nerves injury were also evaluated.

\section{Data Analysis}

The study was conducted using study protocol (Figure 1). All data were statistically analyzed by using Kolmogorov Smirnov and Fischer exact test. 
Irianto et al. / Cross and Lateral Fixations on Supracondylar Humerus Fracture

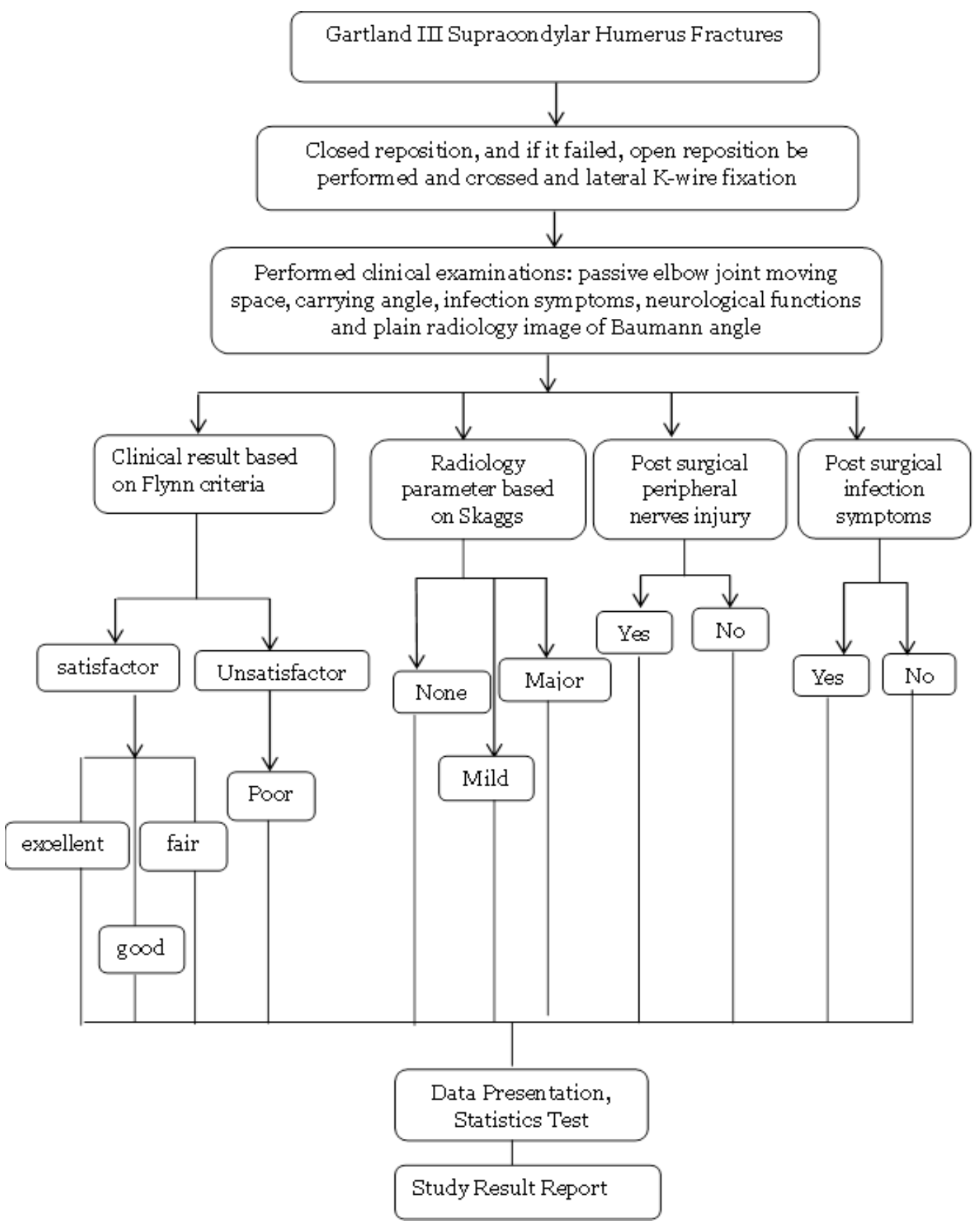

Figure 1. Study Protocol

\section{RESULTS}

The study discovered 28 patients consisted of 20 males and 8 females with the ages ranged from 3 up to 13 years old. The average age in crossed fixation group was 7.6 years and in lateral fixation group was 4.7 years.
The injury sides were right elbow was $46.4 \%$ and left elbow was $53.5 \%$. In crossed fixation group, left elbow injury was $54.5 \%$ and right elbow injury was $45.5 \%$ and in lateral fixation group, left elbow injury was 50\% and right elbow injury was 50\%. 

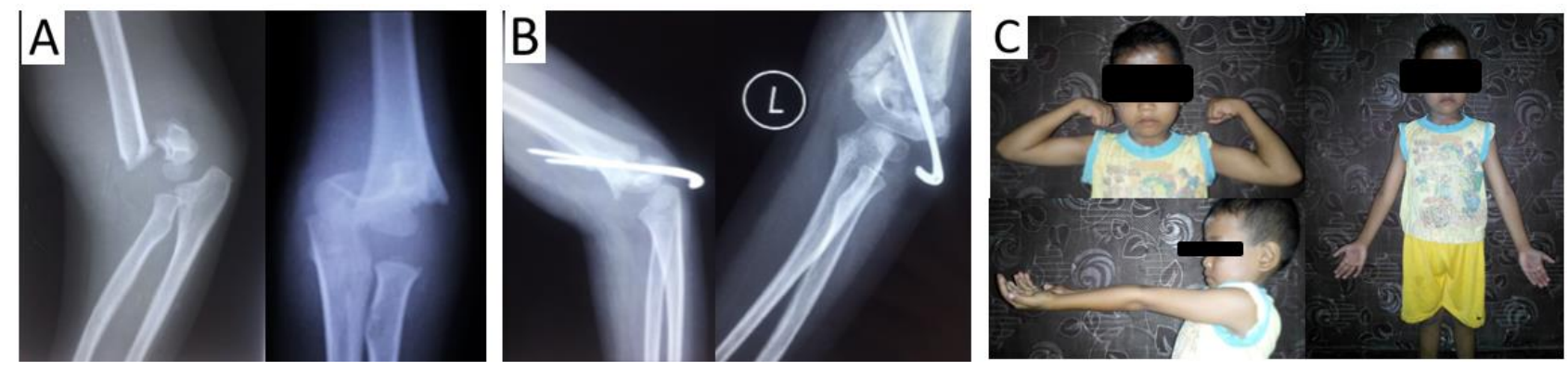

Figure 2. Clinical and Radiology Results of Lateral Fixation of Gartland type III SCHF

(A. Pre-fixation plain image, B. Post-fixation plain image, C. Post-Surgical Clinical Image)
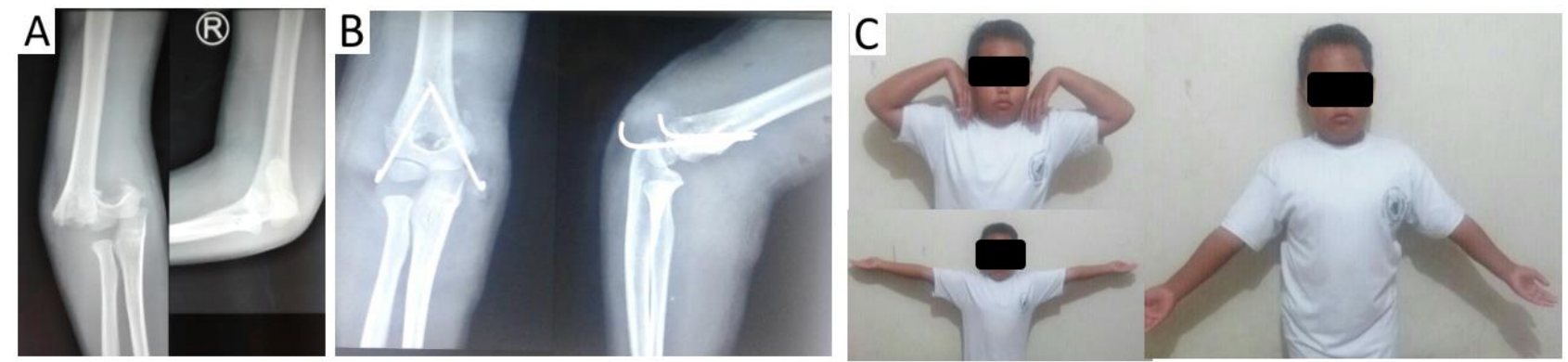

Figure 3. Clinical and Radiology Results of Crossed Fixation of Gartland type III SCHF.

A. Pre-fixation plain image, B. Post-fixation plain image, C. Post-Surgical Clinical Image

The time duration between the incidence of injuries and the surgical procedure spanned from about 5 hours up to 30 hours with the average time was 13.4 hours. It is discovered that the duration between the incidence of injuries and the surgical procedure in crossed fixation was about 5 hours up to 30 hours with the average time was 11.9 hours and in lateral fixation group was about 7 hours up to 26 hours with the average time was 18.7 hours.

Table 1. Satisfactory Degrees measured by using Flynn criteria

\begin{tabular}{lccc}
\hline Satisfactory Degrees & \multicolumn{2}{c}{ Fixation Configurations } & Total \\
\cline { 2 - 3 } & Crossed Fixation & Lateral Fixation & \\
\hline Satisfactory & $\mathbf{2 1}$ & $\mathbf{6}$ & 27 \\
Unsatisfactory & 1 & 0 & 1 \\
Total & 22 & 6 & 28 \\
\hline
\end{tabular}

Satisfactory degree which was measured based on Flynn criteria discovered the satisfactory result on 27 patients and unsatisfactory result on 1 patient. In crossed fixation group it was discovered the satisfactory result on 21 patients and unsatisfactory result on 1 patient, whereas in lateral fixation group it discovered satisfactory result on all 6 patients.

Table 2. Flynn criteria of each treatment group

\begin{tabular}{lccccc}
\hline Treatment & \multicolumn{4}{c}{ Flynn Grading } & Total \\
\cline { 2 - 5 } Groups & Poor & Fair & Good & Excellent & \\
\hline Crossed Fixation & 1 & 3 & 12 & 6 & 22 \\
Lateral Fixation & 0 & 1 & 3 & 2 & 6 \\
total & 1 & 4 & 15 & 8 & 28 \\
\hline
\end{tabular}

The result of data analysis of the study for the result differences of Flynn's satisfactory bet-

ween crossed and lateral fixations by using Fisher Exact Test discover the value of $\mathrm{p}$ was 
Irianto et al. / Cross and Lateral Fixations on Supracondylar Humerus Fracture

0.786 hence, it can be concluded that there is no significant difference between the two treatment groups concerning the resulted clinical functions.

For the differences of clinical functional result based on Flynn criteria which are divided into excellent, good, fair, and poor, an analysis was conducted by using Two-Sample Kolmogorov-Smirnov Test and it discovered the value of $p$ was 1.000 which indicated that there was no significant different between the two treatment groups.

Table 3. Skaggs radiological criteria of each treatment group

\begin{tabular}{lcccc}
\hline Treatment Groups & \multicolumn{3}{c}{ Skaggs Criteria Degree } & \multirow{2}{*}{ Total } \\
\cline { 2 - 4 } & None & Mild & Major & \\
\hline Crossed Fixation & 19 & 2 & 1 & 22 \\
Lateral Fixation & 4 & 2 & 0 & 6 \\
Total & 23 & 4 & 1 & 28 \\
\hline
\end{tabular}

In radiological evaluation measurement based on Skaggs criteria which was divided into none, mild, and major, an analysis by using Two-Sample Kolmogorov-Smirnov Test was conducted and obtained the value of $p$ was 0.993. It indicated that there was no significant different between the two treatment groups. There was no ulnar nerve injury nor post-surgical infections found in all patients both from crossed fixation and lateral fixation groups.

\section{DISCUSSION}

The study discovered male patients were $71.4 \%$ higher in number than female patients which were only $28.6 \%$. The ratio between male vs. female was 2.5:1. The condition is in line with the previous epidemiology study that there were more male patients of supracondylar humerus fractures than female patients with the ratio 2:1.16. It was probably because male had a lot more activities and often conducted activities outside compared with female.

The injuries occurred more on left elbows, whereas all patients in the study were right handed, the injury sides were dominated by non-dominant side (Kasser, 1992). Meanwhile Balakumar and Madhuri (2012) reported $1.1 \%$ ulnar nerve iatrogenic injuries, 2.2\% median nerve injuries and $\mathbf{1 . 1} \%$ radial nerve injuries on both crossed and lateral fixation techniques however, we did not discover peripheral nerve injuries in this study. Awareness and surgeons' adequate skills factors in the surgical process are the crucial factors in avoiding ulnar nerve iatrogenic injury.

There was no significant difference of patients' clinical function result which was measured by using Flynn criteria between crossed and lateral fixation technique groups. Configurations, fixation insertion techniques, the size of K-Wire for fixation, post-surgical rehabilitation, patients' compliance were factors that supported the accomplishment of therapy. Patients' compliance means all patients visits orthopedic polyclinic after the surgery, perform programs from the polyclinics, and obtain adequate rehabilitation therapy.

The study discovered the result of $41 \%$ excellent, $50 \%$ good, $5 \%$ fair and $5 \%$ poor in crossed fixation technique group, meanwhile it discovered the result of $33 \%$ excellent, $50 \%$ good, $17 \%$ fair and $5 \%$ poor in lateral fixation technique group, based on Flynn criteria. It discovered in crossed fixation technique group that the satisfactory result was $92.5 \%$, and the unsatisfactory result was $3.5 \%$, yet in lateral fixation technique group the satisfactory result was $100 \%$. Some previous studies 
Irianto et al. / Cross and Lateral Fixations on Supracondylar Humerus Fracture

discover between $87.5 \%$ up to $100 \%$ of satisfactory result (Davis et al., 2000). This study almost obtained data which are approaching the previous studies.

Based on the radiological evaluation measurement by using Skagg criteria toward crossed fixation and lateral fixation it discovered that there was no significant difference between the two groups $(\mathrm{p}=0.993)$. All patients underwent surgery by using the assistance of fluoroscopy (C-Arm) during the surgery procedure, hence it obtained optimal result of the surgery in term of radiology.

The weakness of the study was insufficient and imbalanced sample between the two therapy groups. It probably would be better if there are more number of sample and the quantity is comparable between the two groups. There is no difference of radiology and clinical functions between the crossed and the lateral fixation reduction technique. The study does not discover the surgical risk in the form of ulnar nerve iatrogenic injury and post-surgical infection.

\section{AUTHOR CONTRIBUTION}

Komang Agung Irianto, Tri Wahyu Martanto, Febrian Brahmana, Laskar PK contributed to the design and implementation of the study, analysis of the results, and writing of the manuscript.

\section{CONFLICT OF INTEREST}

There is no conflict of interest in this study.

\section{FUNDING AND SPONSORSHIP}

This study is self-funded.

\section{ACKNOWLEDGEMENT}

The author would like to say thank you to Dr. Soetomo Hospital, Surabaya, Central Java, Indonesia for allowing this study to be carried out and to all study subjects who were willing and cooperative to be part of this study.

\section{REFERENCE}

Balakumar B, Madhuri V (2012). A retrospective analysis of loss of reduction in operated supracondylar humerus fractures. Indian J Orthop. 46: 6907.

Davis RT, Gorczyca JT, Pugh K (2000). Supracondylar humerus fractures in children. Comparison of operative treatment methods. Clin Orthop Relat Res: 376: 49-55.

Gartland JJ (1959). Management of supracondylar fractures of the humerus in children. Surg Gynecol Obstet: 145154.

Gordon JE, Patton CM, Luhmann SJ, Bassett GS, Schoenecker PL (2001). Fracture stability after pinning of displaced supracondylar distal humerus fractures in children. J Pediatr Orthop, 21(3): 313318

Kalenderer O, Reisoglu A, Surer L, Agus H (2008). How should one treat iatrogenic ulnar injury after closed reduction and per-cutaneous pinning of pediatric supracondylar humeral fractures? Injury, 39(4): 463-466. https://doi.org. 10.1016/j.injury.2007.07.016

Kasser JR (1992). Percutaneous pinning of supracondylar fractures of the humerus. Instr course lect: 41: 385-390.

Kocher MS, Kasser JR, Waters PM, Bae D, Snyder BD, Hresko MT (2007). Lateral entry compared with medial and lateral entry pin fixation for completely displaced supracondylar humeral fractures in children: A randomized clinical trial. J Bone Jt Surg Am, 89(4): 706-712. https://doi.org.10.2106/JBJS.F.o0379

Lee EH (2000). Supracondylar Fractures of the humerus in Children Back to basics. Singapore Med J; 9: 423-424.

Lins RE, Simovitch RW, Waters PM (1999). Pediatric elbow trauma. Orthop Clin North Am, 30:119. https://doi.org.10.1016/s0030-5898(05)70066-3 
Irianto et al. / Cross and Lateral Fixations on Supracondylar Humerus Fracture

Otsuka NY, Kasser JR (1997). Supracondylar fractures of the humerus in children. $\mathrm{J}$ Am Acad Orthop Surg, 5: 19-26. https://doi.org.10.5435/o0124635-199701000-00003

Rasool MN (1998). Ulnar nerve injury after K-wire fixation of supracondylar humerus fractures in children. J Pediatr Orthop, 18: 686-690.

Skaggs DL, Cluck MW, Mostofi A (2004). Lateralentry pin fixation in management of supracondylar fractures in children.
J Bone Joint Surg Am, 86(4): 702-707. https:// doi.org. 10.2106/00004623-200404000-00006

Wang X, Feng C, Wan S, Bian Z, Zhang J, Song M, Shao J, Yang X (2012). Biomechanical analysis of pinning configurations for a supracondylar humerus fracture with coronal medial obliquity. J Pediatr Orthop B, 21: 495-498. https://doi.org.10.1097/BPB.obo13e328355 do1f 